\title{
DIFICULDADES DA ASSISTÊNCIA AO PARTO DOMICILIAR NA ÓTICA DE ENFERMEIRAS OBSTETRAS
}

\author{
DIFFICULTIES IN HOME BIRTH CARE FROM THE \\ PERSPECTIVE OF OBSTETRIC NURSES
}

\section{DIFICULTADES EN LA ATENCIÓN DOMICILIARIA DESDE LA PERSPECTIVA DE LAS ENFERMERAS OBSTETRAS}

\author{
Gabriela dos Santos Pascoto \\ Erika Zambrano Tanaka ${ }^{2}$ \\ Luciane Cristina Rodrigues Fernandes ${ }^{3}$ \\ Antonieta Keiko Kakuda Shimo ${ }^{4}$ \\ Clara Fróes de Oliveira Sanfelice
}

Como citar este artigo: Pascoto GS, Tanaka EZ, Fernandes LCR, Shimo AKK, Sanfelice CFO. Dificuldades da assistência ao parto domiciliar na ótica de enfermeiras obstetras. Rev baiana enferm. 2020;e36633.

Objetivo: investigar as dificuldades encontradas pelas enfermeiras obstetras que estão atuando na assistência ao parto domiciliar. Método: estudo descritivo e de abordagem qualitativa. A coleta de dados foi realizada por meio de uma entrevista semiestruturada com nove enfermeiras obstetras que atendiam partos domiciliares há mais de um ano. Os dados foram analisados segundo a Análise de Conteúdo. Resultados: os depoimentos deram origem a três categorias que revelaram dificuldades relacionadas à: escassez de informações sobre o parto domiciliar; transferência do domicílio para o hospital; e lacunas do processo de trabalho. Conclusão: as enfermeiras obstetras enfrentavam dificuldades de ordem social e prática que fragilizavam e dificultavam a assistência ao parto domiciliar. Estas dificuldades parecem estar relacionadas à falta de regulamentação desse modelo de atenção ao parto nas políticas públicas de saúde do país.

Descritores: Enfermagem Obstétrica. Enfermeiras Obstétricas. Parto Humanizado. Parto Domiciliar. Saúde da Mulher.

Objective: to investigate the difficulties encountered by obstetric nurses who are working in home birth care. Method: descriptive study, with qualitative approach. Data collection was performed through a semi-structured interview with nine obstetric nurses who had been attending home births for more than one year. The data were analyzed according to Content Analysis. Results: the statements gave rise to three categories that revealed difficulties related to: scarcity of information about bome birth; transfer from home to bospital and gaps in the work process. Conclusion: obstetric nurses faced social and practical difficulties that weakened and bindered home birth care. These difficulties seem to be related to the lack of regulation of this model of delivery care in the country's public health policies.

Descriptors: Obstetric Nursing. Nurse Midwives. Humanizing Delivery. Home Childbirth. Women's Health.

\footnotetext{
Enfermeira. Especialista em obstetrícia. Pesquisadora Independente. Campinas, São Paulo, Brasil. http://orcid.org/0000-0002-34I7-87।8.

Enfermeira Obstetra. Mestre em Enfermagem. Doutora em Ciências Médicas. Professora da área de Saúde da Mulher e do Recém-Nascido, Faculdade de Enfermagem, Universidade de Campinas. Campinas, São Paulo, Brasil. http://orcid.org/0000-000 I-99/3-2975.

3 Enfermeira Obstetra. Mestre em Enfermagem. Universidade de Campinas. Campinas, São Paulo, Brasil. http://orcid.org/0000-0002-5535-2383.

4 Enfermeira Obstetra. Mestre e Doutora em Enfermagem. Professora aposentada da Faculdade de Enfermagem da Universidade de Campinas e da Escola de Enfermagem da Universidade de São Paulo. Campinas, São Paulo, Brasil. http://orcid.org/0000-000 I-7377-4590.

Enfermeira Obstetra. Mestre em Enfermagem. Doutora em Ciências da Saúde. Professora da área de Saúde da Mulher e do Recém-Nascido, Faculdade de Enfermagem, Universidade de Campinas. Campinas, São Paulo, Brasil. csanfelice@fenf.unicamp.br. http://orcid.org/0000-0003-1920-3193.
} 
Objetivo: investigar las dificultades encontradas por las enfermeras obstétricas que trabajan en la asistencia al parto domiciliario. Método: estudio descriptivo y de enfoque cualitativo. La recolección de datos se realizó a través de una entrevista semiestructurada con nueve enfermeras obstétricas que realizaban partos domiciliarios durante más de un año. Los datos se analizaron de acuerdo con el Análisis de Contenido. Resultados: de las declaraciones, surgieron tres categorias que revelaron dificultades relacionadas con: escasez de información sobre el parto domiciliario; transferencia de casa al hospital y lagunas en el proceso de trabajo. Conclusión: las enfermeras obstétricas encontraron dificultades sociales y prácticas que debilitaron y obstaculizaron la atención al parto domiciliario. Estas dificultades parecen estar relacionadas con la falta de regulación de este modelo de atención de la prestación en las políticas de salud pública del país.

Descriptores: Enfermeria Obstétrica. Enfermeras Obstétricas. Parto Humanizado. Parto Domiciliario. Salud de la Mujer.

\section{Introdução}

Segundo as Diretrizes Nacionais de Assistência ao Parto Normal do Ministério da Saúde (MS), os profissionais da saúde não devem desencorajar o domicílio como um local de escolha para o parto ${ }^{(1)}$, visto que o parto em casa é uma opção reconhecida pela Organização Mundial da Saúde (OMS) há várias décadas, desde que a assistência seja realizada por profissional qualificado e com plano de transferência estabelecido para os casos indicados ${ }^{(2)}$.

No Brasil, não há nenhuma lei que proíba a realização do parto em domicílio. Na atualidade, esse tipo de assistência, realizado pela obstetriz ou enfermeira obstetra e respaldado pelo Conselho Federal de Enfermagem, é entendido como uma garantia do direito reprodutivo das mulheres ${ }^{(3)}$. Além disso, a OMS, o MS e a última revisão sobre o tema da biblioteca Cochrane recomendam a assistência ao parto de risco habitual pelas enfermeiras obstétricas, uma vez que essas profissionais são amplamente reconhecidas por praticarem uma assistência alinhada com as evidências científicas, a qual se baseia no uso racional e criterioso das intervenções. A assistência prestada pelas enfermeiras obstetras valoriza a fisiologia do processo de parturição, reduz os procedimentos desnecessários e eleva os índices de parto normal e de satisfação materna ${ }^{(1-2,4)}$.

Sobre a temática do parto domiciliar, a literatura atual apresenta estudos bem delineados que apontam para resultados maternos e fetais satisfatórios. Recente revisão sistemática com meta-análise, por exemplo, demonstrou maior taxa de partos espontâneos, menor chances de intervenções e de cirurgia cesariana, menor risco de distócia fetal e hemorragia pós-parto, além de taxas semelhantes para morbimortalidade neonatal quando comparados o ambiente hospitalar e o domiciliar ${ }^{(5)}$.

$\mathrm{Na}$ ótica das mulheres, a literatura aponta a insatisfação com o modelo obstétrico hospitalar vigente como um dos principais motivos de escolha para o parto domiciliar planejado. A assistência intra-hospitalar é vista como um atendimento despersonalizado, baseado em normas e rotinas inflexíveis, que se apropria do momento de vulnerabilidade materna para impor condutas e procedimentos de forma arbitrária, sem considerar o diálogo e as decisões maternas, além de compreender o parto como um processo patológico $^{(6-7)}$.

Diante desse cenário, as estatísticas mostram uma tendência de crescimento nas taxas de parto domiciliar no âmbito nacional e mundial. Nos Estados Unidos, por exemplo, a taxa de parto domiciliar planejado aumentou 77,0\% de 2004 a $2017^{(8)}$. No Brasil, observa-se o mesmo fenômeno nas regiões mais urbanizadas (Sul, Sudeste e Centro-Oeste) no período de 2010 a 2017, sendo a categoria da enfermagem obstétrica a que aderiu de forma majoritária a esse modelo de atendimento ${ }^{(9)}$.

Embora a literatura internacional sobre $\mathrm{O}$ tema seja ampla, uma recente revisão da literatura sobre o parto domiciliar mostra que a produção nacional sobre esta temática ainda é 
escassa, e retrata, principalmente, os desfechos maternos e neonatais deste tipo de assistência ${ }^{(6)}$, além de motivações que justificam a escolha de parir em casa ${ }^{(6-7)}$.

Diante dessa conjuntura, e por se tratar de um campo de atuação da enfermagem obstétrica em franca ascensão no país e com o intuito de suscitar reflexões que possam contribuir com o refinamento e a qualificação dessa modalidade de trabalho, esta pesquisa teve como objetivo investigar as dificuldades encontradas pelas enfermeiras obstetras que estão atuando na assistência ao parto domiciliar.

\section{Método}

Trata-se de uma pesquisa exploratória, descritiva e de abordagem qualitativa. O público alvo deste estudo foram enfermeiras obstetras que trabalhavam na assistência ao parto domiciliar. Foram definidos os seguintes critérios de inclusão: enfermeiras com especialização em obstetrícia; tempo mínimo de um ano de atendimento a partos em domicílio e profissionais atuantes na cidade de Campinas (SP) e região. Foram excluídas da participação enfermeiras obstetras atuantes fora da região delimitada e com menos de um ano de experiência no atendimento ao parto domiciliar planejado.

O número de participantes deste estudo foi definido utilizando-se o conceito de "ponto de saturação", amplamente utilizado em investigações qualitativas. Considera-se que o ponto de saturação é alcançado quando os novos participantes passam a repetir informações que já foram mencionadas em entrevistas anteriores e o acréscimo de novos conteúdos deixa de ser necessário, visto que não altera a compreensão do fenômeno estudado ${ }^{(10)}$.

Os dados foram coletados por meio de um questionário on-line construído pelos autores, com base em um aplicativo gratuito da Internet (Google Forms), composto por duas partes: perfil sociodemográfico das participantes; e entrevista semiestruturada, contendo uma pergunta aberta disparadora: "Você vivenciou/vivencia desafios por assistir partos em domicílio? Se sim, nos conte um pouco sobre eles."

As participantes foram recrutadas pela técnica de amostragem por bola de neve (Snowball Sampling) realizada nos meses de agosto a outubro de 2019. Nesta técnica, os primeiros entrevistados indicam os próximos, que, por sua vez, indicarão outros e assim por diante ${ }^{(11)}$, até o momento em que as novas informações passam a ser repetidas (ponto de saturação) ${ }^{(10)}$. Nesse percurso, 14 enfermeiras obstétricas foram indicadas, mas apenas 9 aceitaram participar da pesquisa. Quando uma recusava-se a participar, solicitava-se da última entrevistada uma nova indicação. Ao alcançar o ponto de saturação, o processo de coleta de dados foi encerrado.

As respostas foram inseridas em planilhas do programa Excel. Os dados foram analisados segundo a Análise de Conteúdo proposta por Bardin, a qual compreende uma técnica de tratamento dos dados que visa obter, por meio de procedimentos sistemáticos, indicadores que permitam a inferência de conhecimentos relativos às mensagens e o desvelamento das relações que se estabelecem além das falas propriamente ditas ${ }^{(12)}$.

A Análise de Conteúdo prevê três fases fundamentais que foram seguidas no tratamento das entrevistas realizadas: pré-análise, exploração do material e tratamento dos resultados (inferência e interpretação) ${ }^{(12)}$.

Visando o rigor metodológico do estudo, utilizou-se o checklist Consolidated Criteria for Reporting Qualitative Studies (COREQ) durante o processo de produção dos dados.

Para garantir o anonimato, as entrevistadas foram identificadas pela letra "E" seguida de um número aleatório. A pesquisa seguiu as normas da Resolução n ${ }^{\circ}$ 466/2012 e da Resolução $n^{\circ}$ 510/2016 do Conselho Nacional de Saúde, sendo conduzida de acordo com os padrões éticos exigidos para pesquisas que envolvem seres humanos.

O estudo foi aprovado pelo Comitê de Ética em Pesquisa, protocolo no $3.437 .075 / 2019$. A anuência e concordância com o Termo de 
Consentimento Livre e Esclarecido foi um pré-requisito para liberação do questionário eletrônico.

\section{Resultados}

Participaram da pesquisa nove parteiras urbanas do sexo feminino com idade entre $27 \mathrm{e}$ 50 anos e média de 36 anos. Todas se autodeclararam de cor branca, três $(33,3 \%)$ eram casadas, sete $(77,8 \%)$ tinham filhos, cinco $(55,6 \%)$ graduaram-se em instituições públicas de ensino, cinco (55,6\%) atuavam há mais de cinco anos na assistência domiciliar e três $(33,3 \%)$ possuíam outro vínculo empregatício concomitante ao atendimento a partos domiciliares.

Dentre as participantes, oito $(88,9 \%)$ afirmaram que, antes de iniciar os atendimentos domiciliares, já atuavam na área de obstetrícia e todas (100\%) afirmaram que pretendiam manter-se na assistência a partos domiciliares pelos próximos cinco anos.

Os depoimentos revelaram as dificuldades vivenciadas por essas profissionais no atendimento ao parto domiciliar, que foram agrupados e deram origem a três categorias temáticas: Escassez de informações sobre o parto domiciliar; Transferência do domicílio para o hospital; e Lacunas do processo de trabalho.

\section{Escassez de informações sobre o parto domiciliar}

Os relatos desta categoria mostraram que as enfermeiras obstetras enfrentavam preconceito por diversas camadas da sociedade em relação à assistência ao parto prestada no domicílio, devido à falta de informações sobre este modelo de assistência:

Primeiro de tudo: a falta de informação pelos próprios profissionais da saúde acerca do assunto; a não aceitação [do parto domiciliar] da maioria dos profissionais de saúde que, por falta de informação, banaliza ou ridiculariza esse modelo de assistência e pior, descreve como irresponsabilidade ou insegurança, sendo que é um modelo de assistência seguro, quando ofertado por profissionais capacitados. (E2).

O maior desafio é o preconceito, tanto da sociedade e da família do casal que opta por isso, quanto das instituições e profissionais de saúde [...] os estereótipos são inúmeros. E assim é visto: parto normal é polêmica; parto domiciliar beira a insanidade. (E1).
O preconceito e os conceitos errôneos estabelecidos na cultura do nosso país sobre o local do parto. (E3).

Isso [o parto domiciliar] não faz parte da cultura brasileira. As pessoas acreditam que nós parteiras temos o objetivo de "causar" dano à mãe e ao bebê. Enquanto o que queremos trazer é segurança e conforto às mulberes que são elegíveis para o parto domiciliar. (E6).

[...] pré-conceito cultural, é uma visão deturpada e equivocada sobre o parto domiciliar. (E7).

Nos culpam por qualquer desfecho negativo. Se a mulher teve hemorragia, a culpa é do parto domiciliar; se o bebê desenvolveu uma sepse neonatal precoce, a culpa também é do parto domiciliar. (E4).

\section{Transferência do domicílio para o bospital}

Os discursos que compõem esta categoria retratam a situação de transferência para o hospital como um grande entrave vivenciado pelas enfermeiras obstetras dentro do processo de trabalho da assistência ao parto em domicílio: A possibilidade de transferência para um hospital é um
estresse para todos. A família será julgada, a equipe será
julgada e muitas vezes ameaçada. Não há um respal-
do para situaçés como essas [transferência hospitalar].
Ficamos à mercê do acaso e da sorte, ou falta dela, sobre
qual profissional nos receberá no hospital e como será
sua atuação e conduta. Em outros países, onde o parto
domiciliar já faz parte da cultura, é possível avisar o hos-
pital mais próximo que um parto está acontecendo nas
redondezase, em caso de transferência, tudo acontece de
forma harmoniosa. (E2).

O grande desafio é quando temos transferências. Os profissionais nos julgam como se estivéssemos fazendo algo ilegal, massacram as mulheres e nos culpam por qualquer desfecho negativo. (E4).

[...] se, por algum motivo, precisamos de uma transferência hospitalar, temos uma resistência e sofremos uma violência extremamente grande que marcam nossas vidas como profissionais... e marcam a vida da família também. (E6).

A pressão que a paciente sofre em ambiente hospitalar, nos casos de transferência, é muito grande. (E1).

Dificuldades nas transferências, pois os profissionais nos recebem mal e tratam mal as gestantes/puérperas. (E5).

Toda vez que transferimos uma paciente, é uma situação delicada, principalmente quando a equipe do hospital sabe que a mulher vem de uma tentativa de parto domiciliar. (E9).

\section{Lacunas do processo de trabalho}

A estrutura de trabalho na assistência ao parto domiciliar apresenta lacunas que foram mencionadas como importantes dificuldades para atuação das enfermeiras obstetras nesse 
contexto. Essas lacunas referem-se à ausência de: serviço que realize exames laboratoriais solicitados pelas enfermeiras obstetras durante o acompanhamento pré-natal ou puerperal; local que permita a compra de insumos básicos (medicações) para uso em ambiente domiciliar pela enfermeira obstetra; e diretrizes/protocolos que direcionem a atuação das enfermeiras obstétricas de forma homogênea. Os depoimentos a seguir são ilustrativos:

Os convênios não aceitam a solicitação de exames pedidos por enfermeira obstetra. (E4).

Temos pouca infraestrutura para realizar a assistência. Dependemos de médicos para, por exemplo, comprar medicações que precisamos usar em casa. (E7).

Não temos um protocolo unificado, que direcione nossas condutas durante o atendimento em casa. Então, cada equipe atende da sua forma... (E8).

\section{Discussão}

Os resultados desta pesquisa apontaram que as enfermeiras obstetras enfrentavam um preconceito de várias camadas da sociedade, inclusive de outros profissionais de saúde, ao prestarem assistência ao parto domiciliar, o qual parece ser fruto da falta de informações consistentes sobre como é realizado o atendimento ao parto em ambiente domiciliar.

Este preconceito pode ser explicado por meio de uma contextualização histórica, já que a sociedade civil e os profissionais de saúde construíram, ao longo das últimas décadas, um paradigma a respeito da assistência ao parto que entende a tecnologia avançada como sinônimo de qualidade e segurança ${ }^{(13)}$. Dessa forma, compreende-se o conflito gerado diante da mudança de um modelo assistencial, que parte de uma assistência tecnicista e intervencionista, para uma prática que resgata o parto natural com pouco ou nenhum uso de aparatos tecnológicos, como é o caso da assistência domiciliar. Sobre este aspecto, foi encontrado um único estudo disponível na literatura, que fosse de encontro ao achado deste estudo, mostrando que, socialmente, o parto domiciliar planejado é percebido como um retrocesso, por negar às mulheres as vantagens do progresso da medicina moderna ${ }^{(13)}$.
Os resultados desta pesquisa mostram que uma parcela da sociedade reconhece o parto domiciliar planejado como um ato de irresponsabilidade e de risco para o binômio, uma vez que as participantes referem ser vítimas de estereótipos e culpabilizações diante de qualquer situação que envolva o parto domiciliar. No entanto, esta interpretação diverge das recentes evidências disponíveis na literatura, as quais demonstram bons desfechos maternos e neonatais, maiores taxas de satisfação materna, menores taxas de intervenção e índices de mortalidade neonatal semelhantes quando comparados os partos em ambiente domiciliar em relação àqueles ocorridos no ambiente hospitalar ${ }^{(6,14-20)}$.

Assim, parece que o atendimento ao parto domiciliar planejado representa um movimento de contracultura na atualidade que sofre todos os estigmas e pré-conceitos de uma profissão que, até o presente momento, não é reconhecida pela sociedade. Entretanto, isso pode ser explicado, pois, segundo as estatísticas atuais, mais de $98,0 \%$ dos partos do Brasil acontecem em instituições de saúde ${ }^{(9)}$, fazendo do ambiente hospitalar um local socialmente reconhecido como de excelência para o nascimento ${ }^{(13)}$. Este cenário reforça a cultura de parto como hospitalar e superestima a segurança que proporciona ${ }^{(13)}$.

O preconceito e a falta de informação sobre o parto domiciliar dificultam o trabalho das enfermeiras obstetras, tornando-as alvo de constante julgamento social. Nesta direção, sugere-se como estratégia de enfrentamento o delineamento de medidas para expansão do conhecimento científico sobre o tema parto domiciliar para o senso comum, em especial a população leiga, o que ampliaria a compreensão dessa modalidade de assistência e poderia auxiliar na desconstrução dos preconceitos sobre a assistência ofertada pelas enfermeiras obstetras. Além disso, sugere-se aumentar a base de conhecimento sobre o parto domiciliar planejado entre todos os provedores de maternidade. As principais temáticas que deveriam ser abordadas incluem critérios para seleção do local de nascimento, o gerenciamento de emergências obstétricas em partos domiciliares planejados, a avaliação crítica da 
literatura sobre segurança do parto em domicílio e a comunicação/colaboração interprofissional quando as mulheres são transferidas de casa para o hospital $^{(21)}$.

Neste sentido, sugere-se também a divulgação de publicações em periódicos científicos, de entrevistas em jornais e o relato em páginas de internet e redes sociais das próprias mulheres que vivenciaram experiências positivas de parto domiciliar, como estratégias para popularizar o conhecimento sobre o tema ${ }^{(14)}$.

Os resultados também mostraram que algumas famílias, por medo do julgamento, preferem não socializar a decisão sobre o parto domiciliar. Tal achado corrobora a literatura atual, que mostra a opção de muitos casais de não expor à sua família e/ou à sociedade a decisão em relação ao lugar onde será o parto, principalmente quando os familiares demonstram preconceito ou resistência a essa escolha ${ }^{(14)}$.

Esta pesquisa mostrou que, até o momento, as enfermeiras obstetras ocupam uma posição desfavorecida no contexto da assistência ao parto domiciliar no Brasil, visto que ainda não conquistaram um espaço socialmente aceito e legitimado para atuar, embora estudos demonstrem um substancial crescimento quantitativo de profissionais de enfermagem que ingressaram nessa modalidade de assistência, sobretudo nas grandes capitais e regiões metropolitanas ${ }^{(6,14)}$.

Por outro lado, o cenário internacional mostra-se mais favorável à atuação das enfermeiras obstetras no parto domiciliar, em especial nos países em que esta é uma modalidade de atendimento integrada ao sistema de saúde local, como é o caso do Canadá ${ }^{(21)}$, Estados Unidos ${ }^{(22)}$ e Holanda ${ }^{(23)}$.

A outra dificuldade que emergiu neste estudo diz respeito à necessidade de transferência do ambiente domiciliar para o hospitalar. Sobre este aspecto, a literatura disponível é vasta, demonstrando taxas de transferências em partos domiciliares que variam de 7 a $24,0 \%{ }^{(15-19)}$. No entanto, percebeu-se escassez de estudos que abordassem o contexto e a qualidade do atendimento na vigência de uma transferência do ambiente domiciliar para o hospitalar, como foi revelado nesta pesquisa.

Os relatos das participantes trouxeram importantes contribuições sobre esse aspecto e descortinaram a violência vivenciada pelas mulheres e pelas equipes de atendimento domiciliar no ambiente hospitalar na ocorrência de uma transferência. Esta situação foi identificada em outro estudo que, diante desta vulnerabilidade, apontou a omissão de algumas famílias quanto à informação da tentativa de parto domiciliar quando chegavam ao hospital, no intuito de evitar julgamentos e retaliações ${ }^{(14)}$.

Os depoimentos desta pesquisa mostraram comportamentos e atitudes de ameaças, retaliações, culpabilizações e tratamento hostil por parte dos profissionais de saúde, os quais são caracterizados, pela literatura, como violência obstétrica ${ }^{(24)}$. Esse fenômeno pode ser o resultado de uma associação de diversos fatores, tais como: ausência de postura ética e respeitosa por parte das equipes hospitalares; falta de informação sobre o processo de trabalho das enfermeiras obstetras no domićlio; invisibilidade, por parte das políticas de saúde do país, desse modelo de trabalho; e ausência de comunicação entre equipes de atendimento e serviços de saúde.

A literatura mostra que diversos países reconhecem a assistência ao parto domiciliar, integrando-os ao sistema de saúde. No Canadá, por exemplo, o parto domiciliar também é assistido por obstetrizes e esta articulação com o sistema de saúde facilita o acesso ao transporte de emergência e à transferência de cuidados obstétricos ou neonatais quando estes são requisitados ${ }^{(16,21)}$. Além disso, a OMS sugere que as parteiras treinadas, regulamentadas, licenciadas e totalmente integradas ao sistema de saúde são capazes de diminuir em $80 \%$ as mortes maternas e neonatais ${ }^{(2)}$, o que justifica a necessidade desta articulação.

No entanto, a ausência de comunicação entre o serviço de referência e o trabalho das enfermeiras obstetras torna invisível a ocorrência de um parto domiciliar. Considera-se que a invisibilidade desse processo de trabalho representa uma séria ameaça à saúde das parturientes e à 
manutenção desta modalidade de assistência, além de atribuir ao atendimento domiciliar, impressões que remetem à irresponsabilidade e/ou clandestinidade. Assim, estudo aponta dois fatores que fragilizam a assistência ao parto domiciliar, que também foram percebidos nos depoimentos desta pesquisa: ausência de reconhecimento deste serviço pelas políticas de saúde e manutenção de um sistema de referência e contrarreferência que não funciona ${ }^{(14)}$.

Diante desse contexto, considera-se importante, no âmbito das políticas públicas de saúde, a determinação de um fluxo de comunicação entre os envolvidos (instituição de saúde e equipe de parto domiciliar em atendimento), para assegurar a possibilidade e a legitimidade da transferência hospitalar. A realização desta transferência, segundo a OMS e o MS, é um dos pré-requisitos para a assistência obstétrica domiciliar segura $^{(1-3,5)}$.

Para tanto, é importante garantir a transferência em tempo oportuno atrelada à recepção hospitalar livre de julgamentos e maus-tratos, para evitar que o acolhimento da parturiente em ambiente hospitalar torne-se conflituoso e violento ${ }^{(14)}$. Assim como mostraram os discursos deste estudo, a literatura também aponta que, ao receber a mulher proveniente de tentativa de parto domiciliar, alguns profissionais de saúde a estigmatizam por ter aderido a uma prática que tecnicamente não é contemplada pelo sistema público de saúde ${ }^{(13)}$ e não é aceita pela opinião pessoal do profissional. Esta ocorrência, entretanto, evidencia uma grave falta de caráter ético, além de caracterizar violação dos direitos humanos.

Diante dos achados desta pesquisa, considera-se importante a inclusão do parto domiciliar planejado nas políticas públicas de saúde do país, para que seja garantida a proteção legal, não somente das mulheres que fazem esta escolha, mas também das enfermeiras obstétricas que estão envolvidas com esta assistência.

A última categoria deste trabalho mostrou-se menos representativa, mas não menos importante. Trouxe à tona as dificuldades práticas vivenciadas no processo de trabalho das enfermeiras obstetras, como a impossibilidade de adquirir insumos básicos para a assistência, de solicitar exames laboratoriais e de imagem, uma das práticas essenciais para determinação do risco gestacional e consequente avaliação da elegibilidade ao parto domiciliar, além da ausência de um protocolo de atendimento que possa direcionar e respaldar as condutas praticadas em ambiente domiciliar.

Segundo a literatura, a utilização de protocolos que padronizem os procedimentos favorece a tomada de decisão do enfermeiro, possibilita corrigir as não conformidades, permite que todos os trabalhadores prestem um cuidado padronizado, de acordo com os princípios técnico-científicos, além de proporcionar maior satisfação e segurança para a equipe de enfermagem e para o paciente ${ }^{(25)}$. Dessa forma, justifica-se a necessidade de elaboração de protocolos e/ou diretrizes que possam guiar e respaldar as práticas assistenciais de atendimento ao parto em domicílio por parte dos órgãos regulamentadores de saúde do país.

No que tange à dificuldade de compra de insumos e de solicitação de exames pelas enfermeiras obstetras que atendem parto domiciliar, não foi encontrada literatura que sustente tais achados, permitindo a interpretação de que esses resultados sejam inéditos.

Considera-se, portanto, que a condição de trabalho exposta limita a assistência ofertada, diminui o grau de autonomia das enfermeiras obstetras no atendimento domiciliar e dificulta o alcance dos melhores resultados. Assim, esta pesquisa mostrou que as fragilidades do processo de trabalho encontradas representam importantes lacunas que precisam ser solucionadas, pois são indispensáveis à atuação segura das enfermeiras obstetras no atendimento ao parto domiciliar.

Ademais, considera-se um equívoco o fato de o MS reconhecer o direito de escolha da mulher sobre o local de parto ${ }^{(1)}$, os órgãos regulamentadores autorizarem a atuação das enfermeiras obstetras em domicílio, incluindo o conselho federal da categoria ${ }^{(3)}$ e, ainda assim, não existir uma estrutura de trabalho desenhada pelas políticas de saúde que torne exequível, de fato, o 
atendimento ao parto domiciliar pelas enfermeiras obstetras.

Dessa forma, reitera-se que a invisibilidade desta modalidade de assistência nas políticas de saúde do país é um fator que dificulta, inclusive, o debate sobre o processo de trabalho que vem sendo praticado pelas enfermeiras obstetras e as possibilidades de qualificá-lo.

Entende-se que os resultados desta pesquisa possuem limitações relacionadas à regionalização da amostra, o que não permite generalizações. Dessa forma, sugere-se a realização de pesquisas futuras que ampliem a amostra para outras regiões do país com o intuito de desvelar (ou reafirmar) as dificuldades encontradas pelas enfermeiras obstetras no atendimento ao parto domiciliar. Além disso, configurou-se como uma limitação para a discussão dos resultados deste estudo a escassez de pesquisas que abordassem as dificuldades vivenciadas nessa modalidade de atendimento ao parto.

\section{Conclusão}

Os resultados do estudo realizado apontam que as enfermeiras obstetras enfrentam importantes dificuldades na trajetória de atendimento ao parto domiciliar planejado, o que inclui o preconceito social com a assistência realizada, gerado pela falta de informação sobre o tema; estigmas e violência obstétrica na ocorrência de uma transferência para o hospital; dificuldades na aquisição de insumos e serviços necessários para a prática domiciliar segura e de qualidade; e ausência de protocolos que direcionem a atuação no domicílio.

Considera-se que estas dificuldades estão relacionadas à invisibilidade do parto domiciliar nas políticas de saúde do país. Conclui-se que as enfermeiras obstetras enfrentam dificuldades de ordem social e prática que fragilizam e dificultam a assistência ao parto domiciliar. Estas dificuldades parecem estar relacionadas à falta de regulamentação desse modelo de atenção ao parto nas políticas públicas de saúde do país.

O reconhecimento das dificuldades enfrentadas pelas enfermeiras obstetras na assistência ao parto domiciliar contribui para dar visibilidade ao trabalho desenvolvido por esta categoria, permitindo a reflexão sobre estratégias de enfrentamento que possam aprimorar e legitimar este modelo assistencial que se encontra em franca expansão na atualidade brasileira.

\section{Colaborações:}

1 - concepção, projeto, análise e interpretação dos dados: Gabriela dos Santos Pascoto, Antonieta Keiko Kakuda Shimo e Clara Fróes de Oliveira Sanfelice;

2 - redação do artigo e revisão crítica relevante do conteúdo intelectual: Gabriela dos Santos Pascoto, Erika Zambrano Tanaka, Luciane Cristina Rodrigues Fernandes e Clara Fróes de Oliveira Sanfelice;

3 - aprovação final da versão a ser publicada: Gabriela dos Santos Pascoto, Antonieta Keiko Kakuda Shimo e Clara Fróes de Oliveira Sanfelice.

\section{Referências}

1. Brasil. Ministério da Saúde. Secretaria de Ciência, Tecnologia e Insumos Estratégicos. Departamento de Gestão e Incorporação de Tecnologias em Saúde. Diretrizes nacionais de assistência ao parto normal: versão resumida [Internet]. Brasília; 2017 [cited 2020 Feb 15]. Available from: https:// bvsms.saude.gov.br/bvs/publicacoes/diretrizes_ nacionais_assistencia_parto_normal.pdf

2. World Health Organization. Strengthening quality midwifery education for Universal Health Coverage 2030: framework for action [Internet]. Geneva; 2019 [cited 2020 Feb 20]. Available from: https://www.who.int/maternal_child_adolescent/ topics/quality-of-care/midwifery/strengtheningmidwifery-education/en/

3. Conselho Federal de Enfermagem. Parecer Técnico CN SM/COFEN № 003/2019, de 14 de agosto de 2019. Dispõe sobre a Regulação e Prática da Enfermagem Obstétrica no espaço do parto domiciliar planejado [Internet]. Brasília; 2019 [cited 2020 Feb 21]. Available from: http:// www.cofen.gov.br/parecer-tecnico-cnsmcofen-no-003-2019_74671.html 
4. Sandall J, Soltani H, Gates S, Shennan A, Devane D. Midwife-led continuity models versus other models of care for childbearing women. Cochrane Database Syst Rev. 2016;4:1-96. DOI: https://doi.org/10.1002/14651858.CD004667.pub5

5. Rossi AC, Prefumo F. Planned home versus planned hospital births in women at low-risk pregnancy: a systematic review with meta-analysis. Eur J Obstet Gynecol Reprod Biol. 2018;222:102-8. DOI: https://doi.org/10.1016/j.ejogrb.2018.01.016

6. Cursino TP, Benincasa M. Parto domiciliar planejado no Brasil: uma revisão sistemática nacional. Ciênc saúde coletiva. 2020;25(4):1433-44. DOI: http://dx.doi.org/10.1590/141381232020254. 13582018

7. Sanfelice CFO, Shimo AKK. Social representations on home birth. Escola Anna Nery. 2015;19(4):606-13. DOI: http://dx.doi.org/10.5935/ $1414-8145.20150081$

8. MacDorman MF, Declercq E. Trends and state variations in out-of-hospital births in the United States, 2004-2017. Birth. 2019;46(2):279-88. DOI: https://doi.org/10.1111/birt.12411

9. Brasil. Ministério da Saúde. Rede Interagencial de Informações para a Saúde. DATASUS. Nascimento por residência mãe por ano do nascimento segundo região/domicílio (2010-2017) [Internet]. Brasília; 2017 [cited 2019 Oct 29]. Available from: http://tabnet.datasus.gov.br/cgi/tabcgi.exe?sinasc/ cnv/nvuf.def

10. Nascimento LCN, Souza TV, Oliveira ICS, Moraes JRMM, Aguiar RCB, Silva LF. Theoretical saturation in qualitative research: an experience report in interview with schoolchildren. Rev Bras Enferm. 2018;71(1):228-33. DOI: https://doi. org/10.1590/0034-7167-2016-0616

11. Naderifar M, Goli H, Ghaljaie F. Snowball Sampling: a purposeful method of sampling in qualitative research. Strides Dev Med Educ. 2017;14(3):e67670. DOI: https://doi.org/10.5812/sdme.67670

12. Bardin L. Análise de conteúdo. São Paulo: Edições 70; 2011.

13. Matão MEL, Miranda DB, Costa BP, Borges TP. A visão médica do parto domiciliar: factível ou utópico? Rev enferm Cent-Oeste Min. 2016;6(2):2147-55.DOI:http://dx.doi.org/10.19175/ recom.v6i2.983

14. Mattos DV, Vandenberghe L, Martins CA. The obstetric nurse in a planned household birth. Rev Enferm UFPE. 2016;10(2):568-75. DOI: 10.5205/reuol.8557-74661-1-SM1002201625

15. Doherty D, Nathan L, Hutchinson M, Somerville S, Hauck Y, Hornbuckle J. Planned home and hospital births in western Australia: 2002-2013. Paediatr Child Health. 2017;53(Suppl. 2):3-17. DOI: https://doi.org/10.1111/jpc.13494_72

16. Hutton EK, Cappelletti A, Reitsma AH, Simioni J, Horne J, McGregor C, et al. Outcomes associated with planned place of birth among women with low-risk pregnancies. CMAJ. 2016;188(5):80-90. DOI: https://doi.org/10.1503/cmaj.150564

17. Santos SS, Boeckmann LMM, Baraldi ACP, Melo MC. Resultados de partos domiciliares planejados assistidos por enfermeiras obstétricas. Rev Enferm UFSM. 2018;8(1):129-43. DOI: http:// dx.doi.org/10.5902/2179769228345

18. Koettker JG, Brüggemann OM, Knobel R. Maternal results from planned home births assisted by nurses from the Hanami team in the south of Brazil, 2002-2012. Texto Contexto Enferm. 2017;26(1):e3110015. DOI: http://dx.doi. org/10.1590/0104-07072017003110015

19. Blix E, Kumle MH, Ingversen $\mathrm{K}$, Huitfeldt AS, Hegaard HK, Ólafsdóttir ÓÁ, et al. Transfers to hospital in planned home birth in four Nordic countries: a prospective cohort study. Acta Obstet Gynecol Scand. 2016;95(4):420-8. DOI: https://doi. org/10.1111/aogs.12858

20. Jonge A, Geerts CC, Goes BYV, Mol BW, Buitendijk SE, Nijhuis JG. Perinatal mortality and morbidity up to 28 days after birth among 743070 low-risk planned home and hospital births: a cohort study based on three merged national perinatal databases. BJOG. 2015;122(5):720-8. DOI: 10.1111 / 1471-0528.13084

21. Vedam S, Stoll K, Schummers L, Fairbrother N, Klein MC, Thordarson D, et al. The Canadian birth place study: examining maternity care provider attitudes and interprofessional conflict around planned home birth. BMC Pregnancy Childbirth. 2014;14:353. DOI: https://doi.org/10. 1186/1471-2393-14-353

22. Cook E, Avery M, Frisvold M. Formulating evidencebased guidelines for certified nurse-midwives and certified midwives attending home births. J Midwifery Womens Health. 2014;59(2):153-9. DOI: https://doi.org/10.1111/jmwh.12142

23. Geerts CC, Klomp T, Janssen ALML, Twisk JWR, Dillen J, Jonge A. Birth setting, transfer and maternal 
sense of control: results from the DELIVER study. BMC Pregnancy Childbirth. 2014;14:27. DOI: https://doi.org/10.1186/1471-2393-14-27

24. Betron ML, McClair TL, Currie S, Banerjee J. Expanding the agenda for addressing mistreatment in maternity care: a mapping review and gender analysis. Reprod Health. 2018;15(1):143-56. DOI: https://doi.org/10.1186/s12978-018-0584-6
25. Sales CB, Bernardes A, Gabriel CS, Brito MFP, Moura AA, Zanetti ACB. Protocolos Operacionais Padrão na prática profissional da enfermagem: utilização, fragilidades e potencialidades. Rev Bras Enferm. 2018;71(1):126-34. DOI: https://doi. org/10.1590/0034-7167-2016-0621

Recebido: 4 de maio de 2020

Aprovado: 13 de julho de 2020

Publicado: 30 de setembro de 2020

A Revista Baiana de Enfermagem utiliza a Licença Creative Commons - Atribuição-NãoComercial 4.0 Internacional.

https://creativecommons.org/licenses/by-nc/4.0/

Este artigo é de acesso aberto distribuído sob os termos da Licença Creative Commons (CC BY-NC).

Esta licença permite que outros remixem, adaptem e criem a partir do seu trabalho para fins não comerciais. Embora os novos trabalhos tenham de lhe atribuir o devido crédito e não possam ser usados para fins comerciais, os usuários não têm de licenciar esses trabalhos derivados sob os mesmos termos. 